\title{
Appendix B-1
}

\# $\mathrm{R}$ code to calculate the approximate group sizes for testing non-inferiority/

\# superiority of a linear contrast for a designated power

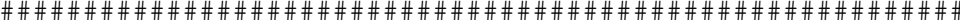

\# 1. You need to specify the following values in the input part: significance level,

\# Type II error, the deviation from $\mathrm{H}_{0}$ to $\mathrm{H}_{a}$, contrast coefficients,

\# and the corresponding unit cost, and variance for each group.

\# 2. Based on different purposes, you can have different group size allocation ratios.

\# You can choose gamma=1 to minimize total cost (MC), gamma=2 to minimize total

\# sample size (MS) or gamma=3 for equal group size (ES).

\# 3. We use the data from Clarke et al. (2005) and the sample size calculation is

\# demonstrated in the Section of illustrative example in this paper.

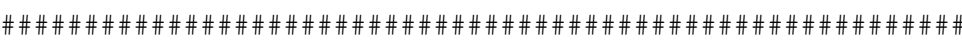

\# begin of the function of SizeInfe

SizeInfe = function(alpha, beta, Delta, lc, cost, variance, gamma)

\# Step 1: Calculate the group size allocation ratio and the initial group sizes

\# Equ.11 of allocation ratio for minimal total cost

$\operatorname{gam} 1=\operatorname{abs}(1 \mathrm{c} / 1 \mathrm{c}[1]) *(\operatorname{variance} / \operatorname{variance}[1]) \wedge 0.5 *(\operatorname{cost}[1] / \operatorname{cost}) \wedge 0.5$

gam2 $=\operatorname{abs}\left(1 \mathrm{c} / \mathbf{l c}_{[1]}\right)^{*}($ variance/variance[1])^0.5 \# for minimal sample size

gam3 $=c(\operatorname{rep}(1$, length $(1 c))) \quad$ \# for equal group size

if $($ gamma $==1) \quad\{$ gam $=$ gam 1$\}$

else if (gamma $==2) \quad\{$ gam $=$ gam2

else $\{$ gam $=$ gam3 $\}$

zalpha = qnorm(1-alpha) \# quantile of the standard normal distribution

zbeta $=$ qnorm $(1-$ beta $)$

sum0 $=1 c^{\wedge} 2 \% *$ (variance/gam $)$

zn1 $=\operatorname{sum0*}($ zalpha+zbeta)^2/Delta^2 \# initial first group size in Equ. 2

$\mathrm{zn}=\mathrm{zn} 1 *$ gam \# initial sizes for all groups

\# Step 2: Calculate the degrees of freedom and find the quantile of the t distribution

$\mathrm{df}=$ function(lc, variance, ns) \# find degrees of freedom in Equ. 14

\{

$\mathrm{u} 1=1 \mathrm{c}^{\wedge} 2 *$ variance / ns

$\operatorname{sum} 1=\operatorname{sum}(u 1)$

$\operatorname{sum} 2=\operatorname{sum}\left(u 1^{\wedge} 2 /(\mathrm{ns}+1)\right)$

$\mathrm{df}=\operatorname{sum}^{\wedge} 2 / \operatorname{sum} 2-2$

\}

$\mathrm{dfl}=\mathrm{df}\left(\mathrm{lc}_{\mathrm{c}}\right.$ variance, $\left.\mathrm{zn}\right)$ 


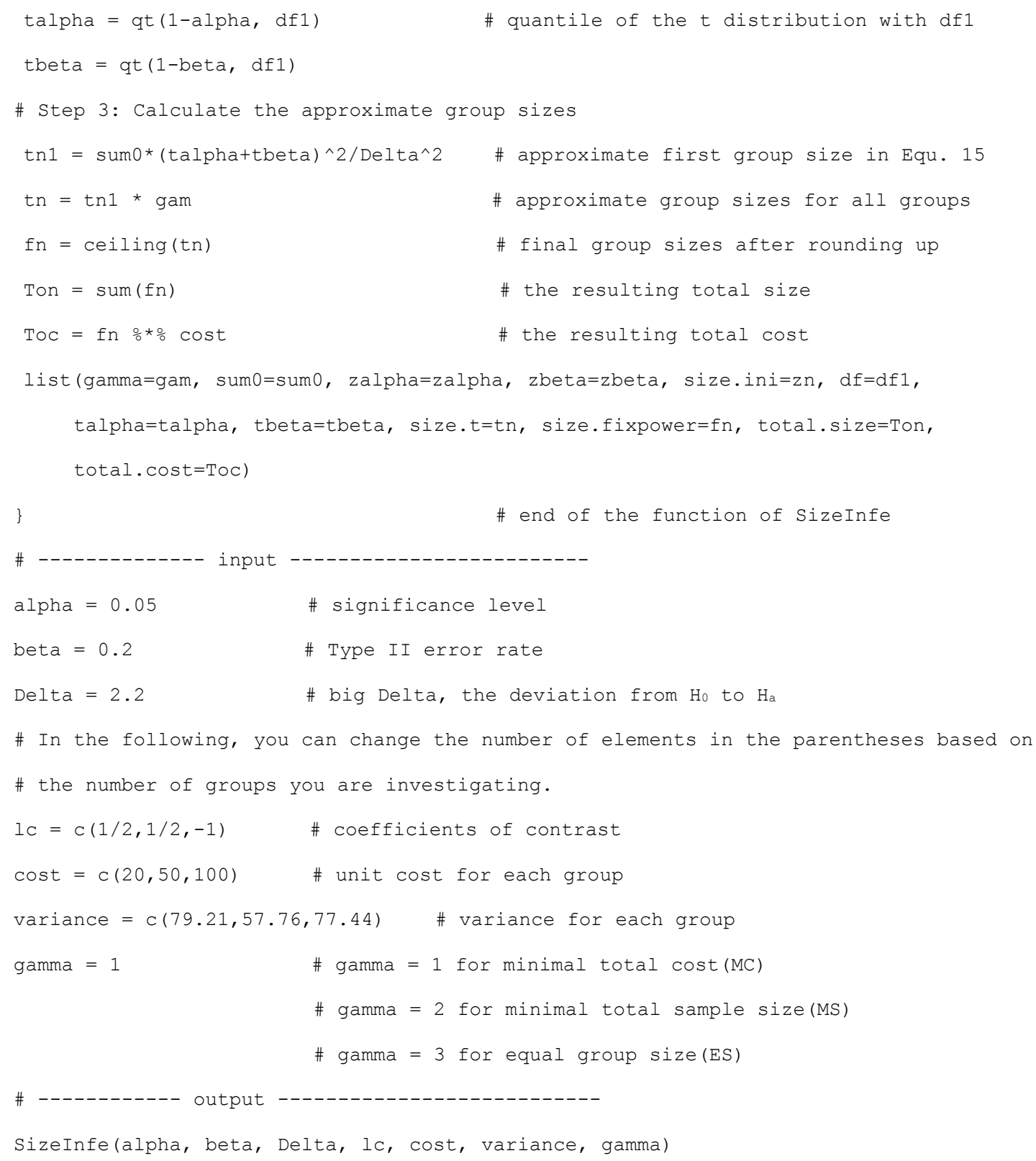




\section{Appendix B-2}

\# $\mathrm{R}$ code to calculate the approximate group sizes for testing equivalence of a linear \# contrast for a designated power

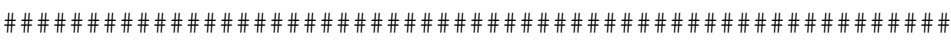

\# 1. You need to specify the following values in the input part: significance level, \# Type II error, equivalence margins (upper \& lower), linear contrast for the

\# alternative hypothesis, contrast coefficients, and the corresponding unit cost,

\# and variance for each group.

\# 2. Based on different purposes, you can have different group size allocation ratios.

\# You can choose gamma=1 to minimize total cost (MC), gamma=2 to minimize total

\# sample size (MS) or gamma=3 for equal group size (ES).

\# 3. We use the data from Van Wier et al.(2012) and the sample size calculation is

\# demonstrated in the section of illustrative example in this paper.

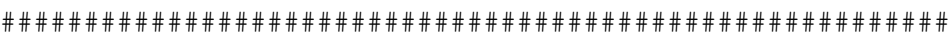

\#------------------- procedure

\# begin of the function of SizeEqui

SizeEqui = function(alpha, beta, deltal, delta2, delta, lc, cost, variance, gamma)

\# Step 1: Calculate the group size allocation ratios and the initial group sizes

\# Equ. 11 of allocation ratio for minimal total cost

$\operatorname{gam} 1=\operatorname{abs}\left(1 \mathrm{c} / I_{\mathrm{c}}[1]\right) *(\operatorname{variance} / \operatorname{variance}[1]) \wedge 0.5 *(\operatorname{cost}[1] / \operatorname{cost}) \wedge 0.5$

gam2 = abs (lc/lc[1])*(variance/variance[1])^0.5 \# for minimal sample size

gam3 $=\mathrm{c}(\operatorname{rep}(1$, length $(1 \mathrm{c})))$

\# for equal group size

if $($ gamma $==1) \quad\{$ gam $=$ gam1 $\}$

else if (gamma $==2) \quad\{$ gam $=$ gam2 $\}$

else $\{$ gam $=$ gam3 $\}$

zalpha $=$ qnorm $(1-a l p h a)$

\# quantile of the standard normal distribution

if $($ delta $==($ delta $1+\operatorname{delta}) / 2) \quad$ power $=1$-beta $/$

\# given power in Equ. 8

\} else $\{$ power $=1$-beta $\}$

\# given power in Equ. 9

zbeta $=$ qnorm (power)

sum0 $=1 \mathrm{C}^{\wedge} 2 \div * \%($ variance/gam $)$

eta $=\min ($ delta - deltal, delta 2 - delta $)$

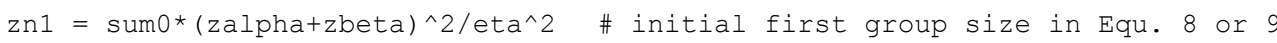

$\mathrm{zn}=\mathrm{zn} 1 *$ gam \# initial sizes for all groups

\# Step 2: Calculate the degrees of freedom and find the quantile of the t distribution

$\mathrm{df}=$ function(lc, variance, ns) \# find degrees of freedom in Equ. 14

$\mathrm{u} 1=\mathrm{lc}^{\wedge} 2 *$ variance / ns

sum1 = sum(u1) 


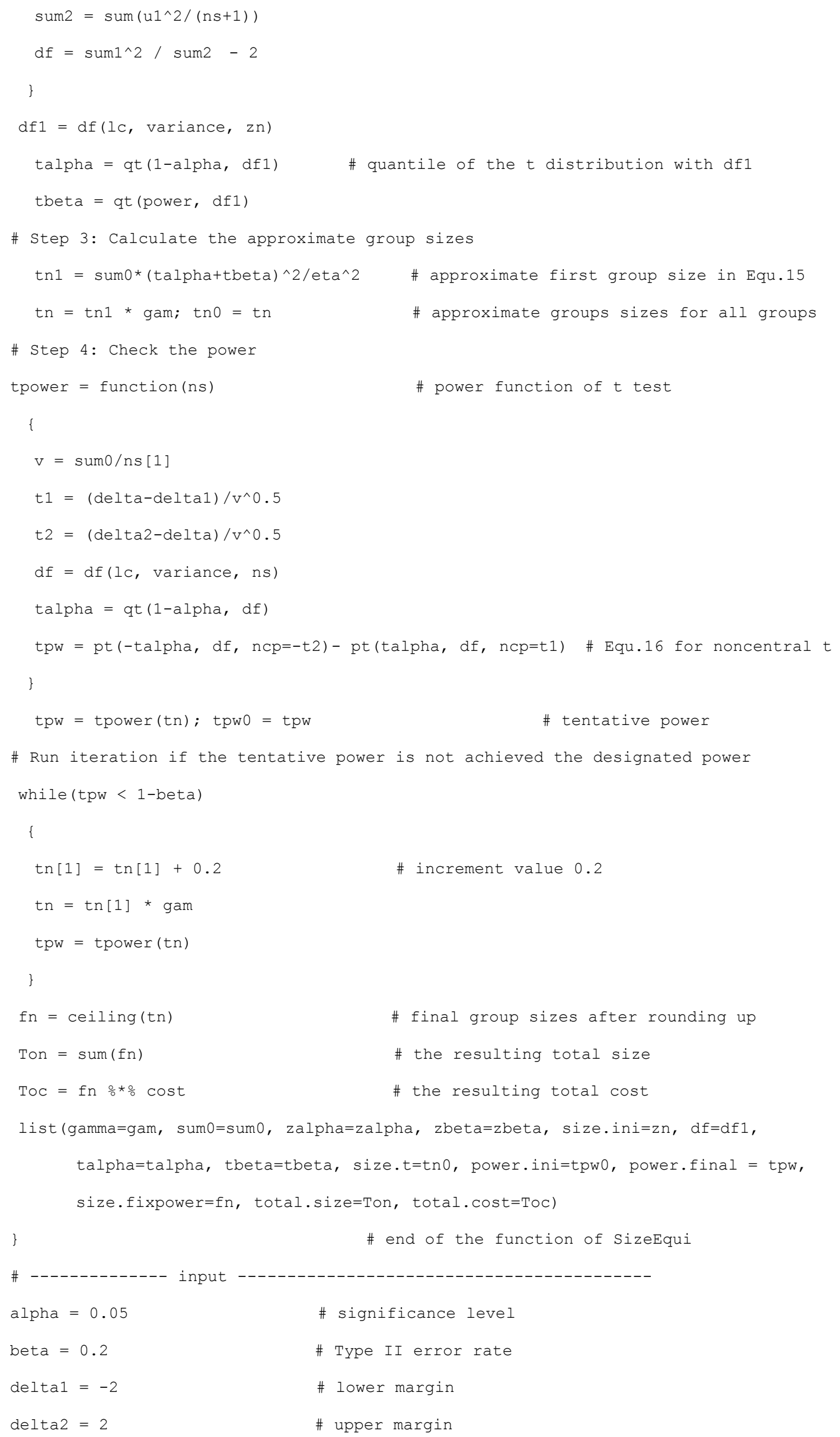


delta $=0.2$

\# linear contrast for the alternative hypothesis

\# In the following, you can change the number of elements in the parentheses based on \# the number of groups you are investigating.

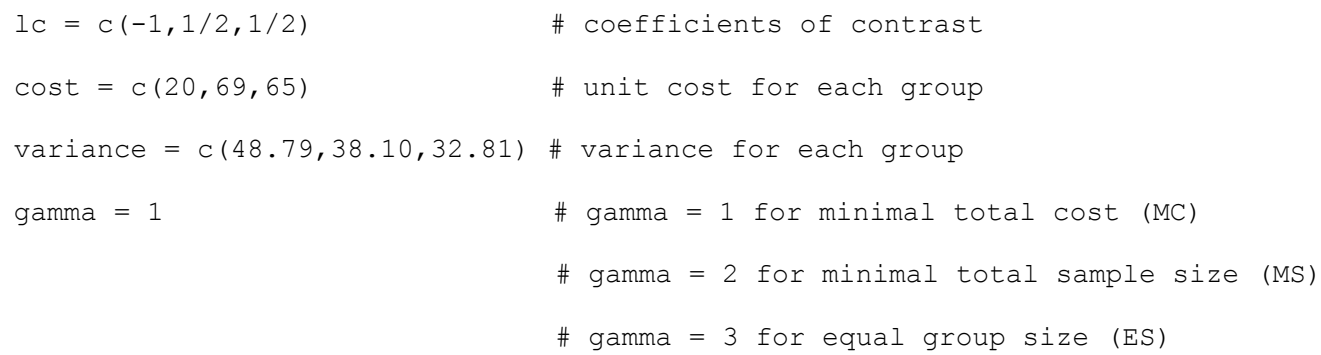

SizeEqui(alpha, beta, deltal, delta2, delta, lc, cost, variance, gamma) 


\section{Appendix B-3}

\# R code to calculate other non-fixed group sizes when some group sizes are fixed, \# for testing equivalence of a linear contrast for a designated power

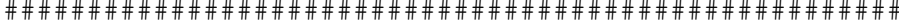

\# 1. You need to specify the following values in the input part: significance level, \# Type II error, equivalence margins (upper \& lower) and linear contrast for the

\# alternative hypothesis. You also need to specify the vectors of contrast

\# coefficients of fixed group(s) and non-fixed group(s), the corresponding

\# variances and unit costs, respectively. (see Input statement)

\# 2. Based on different purposes, you can have different group size allocation ratios.

\# You can choose gamma=1 to minimize total cost (MC), gamma=2 to minimize total

\# sample size (MS) or gamma=3 for equal non-fixed group size (ES).

\# 3. We use the data from Van wier et al.(2012) and the sample size calculation is \# demonstrated in the Section of illustrative example in this paper.

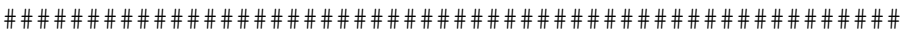

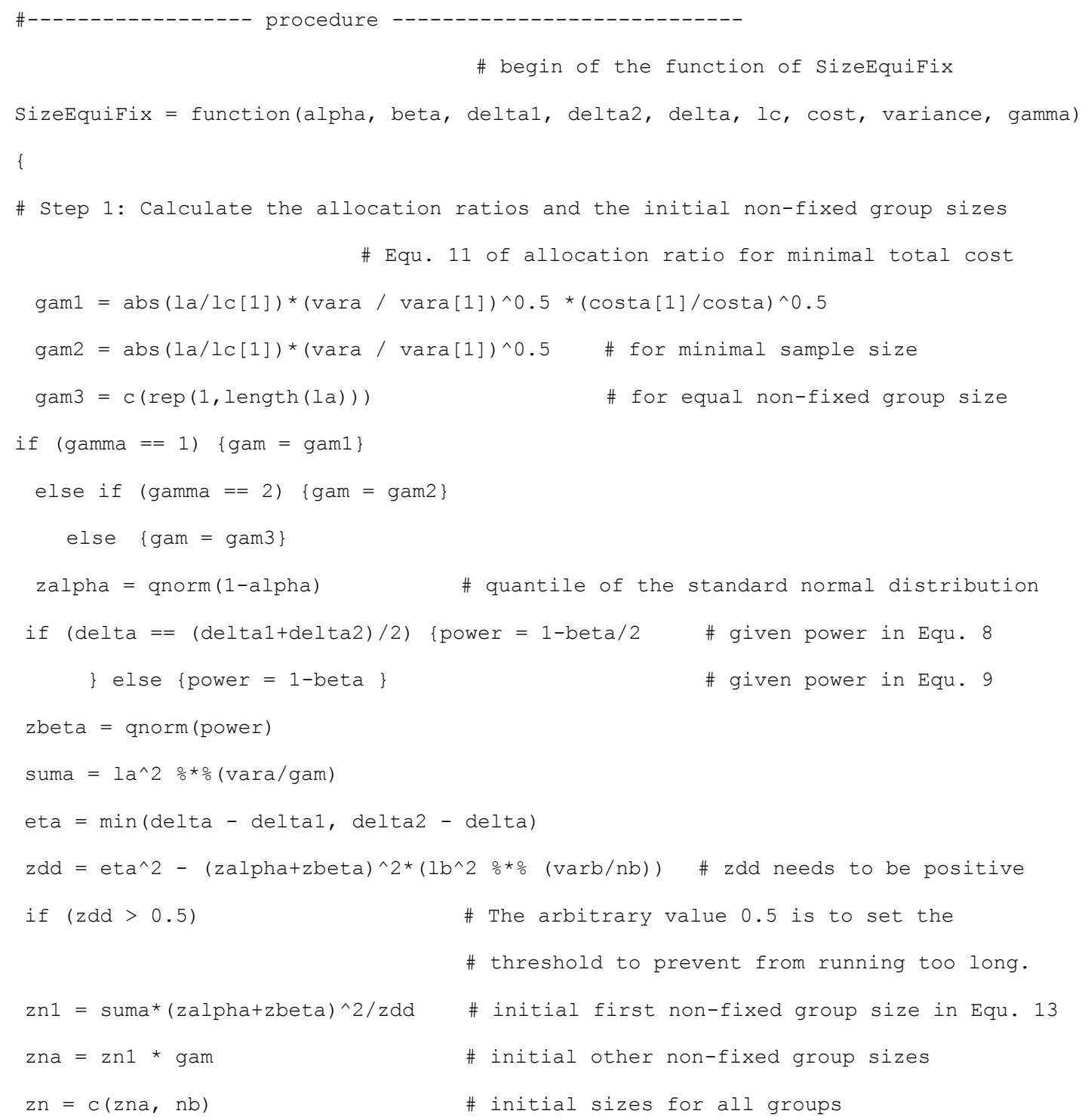




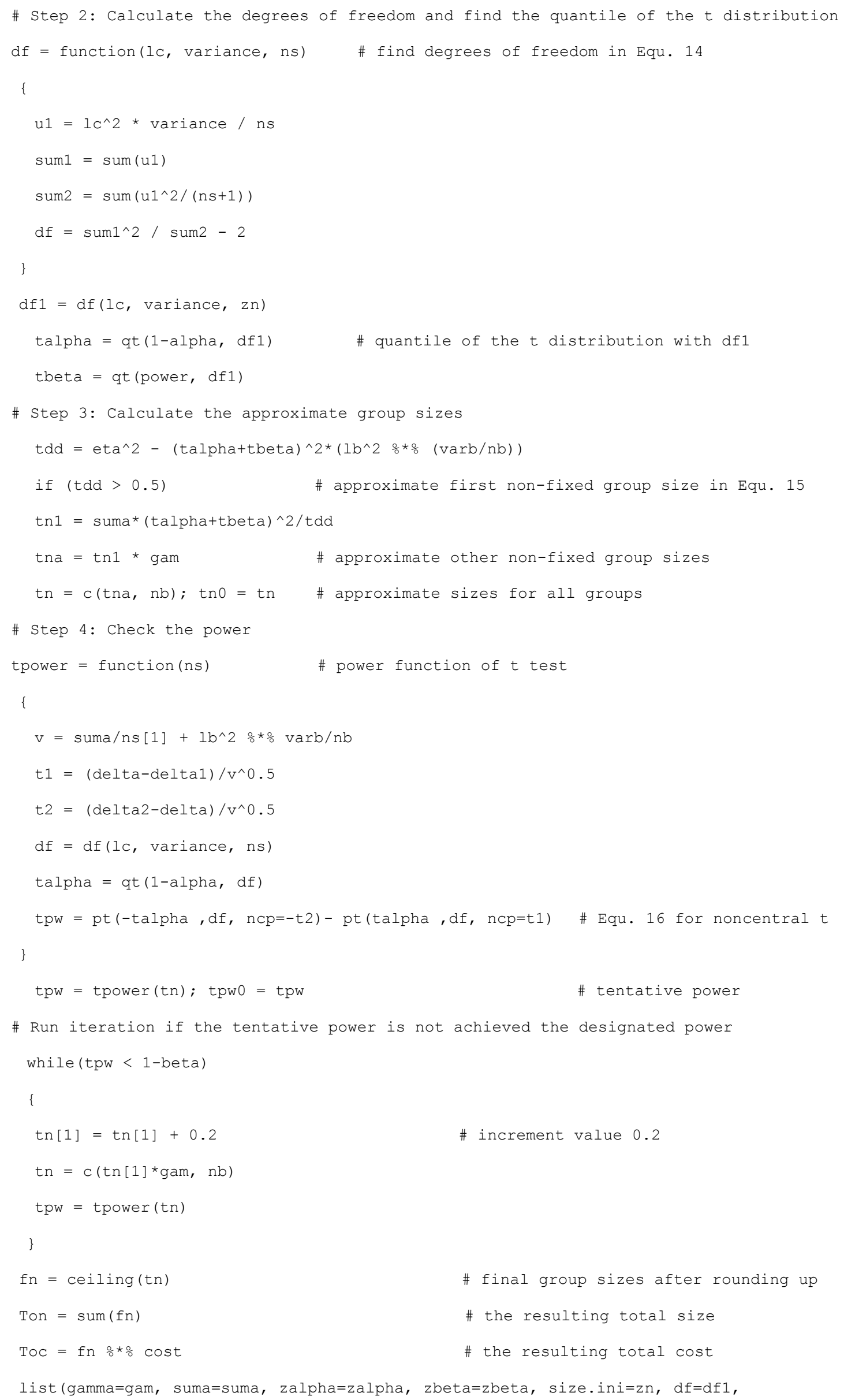




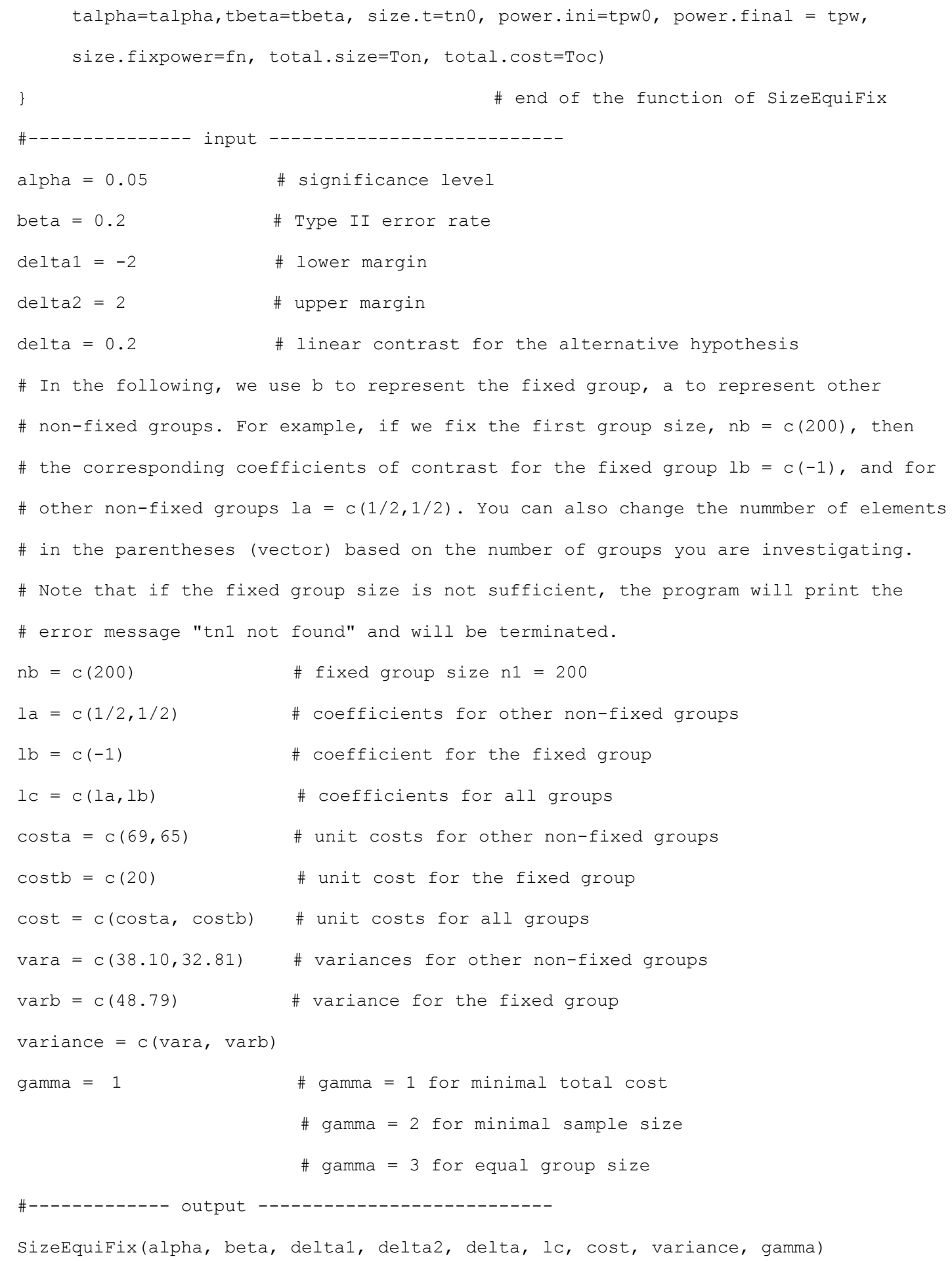

